\title{
Rotavirus Disease in Germany-A Prospective Survey of Very Severe Cases
}

\author{
Sonu Shai, * Ruy Perez-Becker, * Carl-Heinz Wirsing von König, MD, † Rüdiger von Kries, MD, + Ulrich Heininger, MD, $\S$ \\ Johannes Forster, MD, $\$ Hans-Iko Huppertz, MD, Reinhard Roos, MD, ** Ulrich Göbel, MD, †† and Tim Niehues, MD*
}

\begin{abstract}
Objective: Rotavirus (RV) gastroenteritis is a notifiable disease in Germany. The reports to the authorities contain few data concerning the severity of disease. The aims of this study were to determine incidence and outcome of very severe cases of RV disease.

Methods: Cases of very severe RV disease were collected by the German Paediatric Surveillance Unit for rare diseases (Erhebungseinheit für seltene pädiatrische Erkrankungen in Deutschland) using anonymous questionnaires based on hospitalized patients between April 2009 and March 2011. Inclusion criteria were detection of RV antigen in feces, patient aged $0-16$ years and 1 or more of the following criteria: intensive care treatment, hypernatremia or hyponatremia ( $>155 \mathrm{mmol} / \mathrm{L}$ or $<125 \mathrm{mmol} / \mathrm{L}$ ), clinical signs of encephalopathy (somnolence, seizures, apnea) and RV-associated death.
\end{abstract}

Results: During 2 years, 130 cases of very severe RV disease were reported, 101 of 130 were verified. Seventeen patients had nosocomial infection, of whom 14 were neonates in intensive care. Among those, 12 infants had verified or suspected necrotizing enterocolitis. Eighty-four communityacquired cases were reported, median age was 10.5 months ( $0-108$ months). The median hospital stay was 6 days, and 48 patients needed intensive care treatment. Among children less than 5 years of age, the yearly incidence of community-acquired very severe RV disease was 1.2 of 100,000 (95\% confidence interval: $0.9-1.4 / 100,000)$. A total of 26 of 84 and 10 of 84 patients had severe hypernatremia or hyponatremia, respectively, and 58 of 84 patients had signs of encephalopathy. Three deaths were reported (1 nosocomial and 2 community acquired).

Conclusions: RV infection in Germany can have a life-threatening course. A substantial number are nosocomial infections.

Key Words: rotavirus, severe prospective epidemiology, Germany

(Pediatr Infect Dis J 2013;32: e62-e67)

Accepted for publication August 15, 2012.

From the *Zentrum für Kinder- und Jugendmedizin, HELIOS Klinikum Krefeld; $†$ Institut für Hygiene und Laboratoriumsmedizin, HELIOS Klinikum Krefeld, Krefeld; $¥$ Institut für Soziale Pädiatrie und Jugendmedizin, LudwigMaximilians-Universität München, Munich; §Universitäts-Kinderspital beider Basel, Basel, Schweiz; $\uparrow$ Abteilung für Kinder und Jugendmedizin mit Neonatologie, St. Josefskrankenhaus Freiburg im Breisgau; |Klinikum Bremen-Mitte, Bremen, Germany; **München; ††ESPED, Universitätsklinikum Düsseldorf, Düsseldorf, Germany.

The fees (5000 Euro) for the case query through ESPED and collection of the monthly reports during the 2-year study period were covered by GlaxoSmithKline (GSK; München,Germany). None of the authors received personal grants or funding for this study. JF has been a member of the advisory board of GSK Germany on rotavirus vaccine and had travel expenses paid by GSK and Pasteur to give a talk on rotavirus epidemiology. UH is a member of and has been a consultant to the independent Data Monitoring Board for GSK, Rixensart, Belgium, and has received payment for lectures by most major vaccine manufacturer. H-IH receives payments by GSK and Sanofi-MSD for lectures. RR was a member of the advisory board on rotavirus vaccine by GSK. C-HWvK has received payments by GSK for lectures. RvK's institution receives grants. The authors have no other funding or conflicts of interest to disclose.

Address for correspondence: Sonu Shai, HELIOS Klinikum Krefeld, Zentrum für Kinder- und Jugendmedizin, Lutherplatz 40, 47805 Krefeld, Germany. E-mail: sonu.shai@helios-kliniken.de.

Copyright $(C 2013$ by Lippincott Williams \& Wilkins

ISSN: 0891-3668/13/3202-00e62

DOI: 10.1097/INF.0b013e31826f602b
$\mathrm{R}$ otavirus (RV) is the most common cause of gastroenteritis (GE) in children in Germany ${ }^{1}$ and worldwide. ${ }^{2}$ It is estimated that in a global setting, RV disease annually causes about 530,000 deaths in children less than 5 years of age. ${ }^{3}$ However, in industrialized countries, RV GE is usually a mild and self-limiting disease. Nevertheless, it is estimated that in the European Union more than 87,000 hospitalizations and 231 deaths per year are attributed to RV in children less than 5 years of age. ${ }^{4}$ With an estimated annual 3.6 million cases of RV GE in children less than 5 years of age in the European Union, RV causes a heavy burden on society and the healthcare system. ${ }^{4}$ Since 2001 , acute RV disease with either diarrhea and/or vomiting in Germany is to be reported to the Robert Koch Institute (Berlin, Germany), the German federal institution responsible for disease control and prevention. However, due to underreporting, the true number of cases is probably 5-10 times higher than the reported cases. ${ }^{5}$

The 2 available vaccines against RV are safe and effective. ${ }^{6-8}$ In 2009, the World Health Organization recommended global use of RV vaccines. ${ }^{9} \mathrm{RV}$ immunization is not part of the national vaccination program in Germany but is under consideration by the German Standing Committee on Vaccination. ${ }^{1}$ In the absence of a national recommendation for RV vaccination, some individual federal states have still recommended RV vaccination. The RV vaccine is thus used on a limited level in Germany.

Very severe courses of RV infections associated with clinical features such as encephalopathy, severe electrolyte disturbance, intensive care treatment and death have been described. ${ }^{10,11}$ Information on very severe RV disease (vsRVd) is primarily based on case reports or retrospective analyses, whereas prospectively collected data are lacking. Especially, prospective studies to query the vsRVd are not available.

This study aimed to prospectively detect all vsRVd in children younger than 17 years in Germany in a 2-year period, to gather demographic and incidence data as well as details about each individual case. The data could help to better understand the virulent properties of RV and could inform decision on a national $\mathrm{RV}$ vaccine recommendation.

\section{METHODS}

Prospective data collection was coordinated by the wellestablished German Paediatric Surveillance Unit for rare diseases (ESPED; Erhebungseinheit für seltene pädiatrische Erkrankungen in Deutschland). Data on the reliability of the ESPED tool have been published previously. ${ }^{12-14}$ Briefly, ESPED sends a report card to each pediatric department in Germany asking pediatricians for incident diagnoses with specific inclusion criteria of up to 12 conditions on a monthly basis. Each condition is investigated by a principal investigator. Report cards are to be returned to ESPED even if no incident diagnosis is reported. Reports of incident diagnoses are forwarded to the respective principal investigator. If an incident diagnosis is reported, the reporting department is asked to complete a questionnaire that is returned to the principal investigator to collect the relevant data. The current return rate of the report cards to ESPED is more than 95\%. ${ }^{15}$ Between April 2009 and March 2011, 
vsRVds were collected on the monthly report cards. The case definition was patient $0-16$ years of age, detection of RV in feces and a very severe course of RV disease defined by 1 or more of the following criteria: intensive care treatment, hypernatremia $>155$ $\mathrm{mmol} / \mathrm{L}$, hyponatremia $<125 \mathrm{mmol} / \mathrm{L}$, clinical signs of encephalopathy (somnolence, seizures and apneas) and death due to complications related to RV disease.

Patients with a nosocomial RV disease already in intensive care treatment were also included because the underlying diseases for admission to the intensive care unit (ICU) might increase the severity of the RV disease. Once a case was reported, an anonymous questionnaire was sent to the reporting clinic and an anonymized medical discharge report was asked for. Through the questionnaires, basic demographic data were acquired in addition to data related to RV disease such as preadmission duration of symptoms, $\mathrm{RV}$ vaccine status, clinical and laboratory findings, therapy, duration of hospital stay and health status on discharge. RV disease was classified as nosocomial infection if onset of RV symptoms occurred more than 48 hours after hospital admission. ${ }^{1}$

The population number of children and adolescents between 0 and 16 years of age in Germany was $12,636,179$ and 12,515,138 in 2009 and 2010, respectively, adding up to a total of $25,151,317$ of whom $6,818,728$ were less than 5 years of age and 2,701,794 were less than 2 years of age (German Federal Statistical Officehttps://www-genesis.destatis.de; accessed April 27 2012). Population data for 2011 were not available at the time of data retrieval.

Collected data were continuously entered into LibreOffice Calc (version 3.4.4). Data were imported to and analyzed using $\mathrm{R}$ (version 2.14.1), a language and environment for statistical computing. ${ }^{16}$ Medians with interquartile ranges (IQRs: $25-75$ percentile), complete ranges and means where appropriate were used for descriptive analysis. For comparison of categorical variables Fisher exact test was used. For assessing continuous variables, the MannWhitney-Wilcoxon test was applied because parametric nature of the variables could not be assumed. Comparison of categorical variables with an assumed or estimated probability distribution was performed with Pearson $\chi^{2}$ test (Goodness of Fit). The Poisson distribution was used to calculate $95 \%$ confidence intervals (CIs) of count data. Level of significance was set to $P<0.05$. The study protocol was approved by the ethics committee of the University of Essen, Essen, Germany.

\section{RESULTS}

Between April 2009 and March 2011, 130 cases of vsRVd were reported. A completed questionnaire or written or oral feedback could not be retrieved in 10 cases despite multiple enquiries, yielding a questionnaire return rate of $92 \%$. After deducting reports of patients not fulfilling the inclusion criteria (18 reports) and 1 double report, 101 cases were evaluable from 58 different pediatric departments in Germany. The majority of reports $(96 / 101$; $96 \%$ ) concerned children less than 5 years of age. There were 17 nosocomial and 84 community-acquired vsRVd, none of which had occurred in a child who had received an RV vaccine.

\section{Incidence}

To estimate the yearly incidence of vsRVd with a $95 \% \mathrm{CI}$, only community-acquired cases were taken into account as the reports of nosocomial disease cannot be regarded as independent occurrences (eg, multiple cases within 1 clinic). There were 79 reports of community-acquired vsRVd in children under the age of 5 years yielding an incidence of 1.2/100,000/year in children less than 5 years of age; $95 \%$ CI: $0.9-1.4 / 100,000$. Considering patients less than 2 years of age with a community-acquired disease (65 patients), the yearly incidence was $2.4 / 100,000 /$ year; 95\% CI: $1.9-3.1 / 100,000$.

\section{Seasonal Variation}

The monthly incidence of vsRVd varied in parallel to the total number of reported RV infections in children less than 14 years of age reported to the Robert Koch Institute as seen in Figure 1. Yearly peak incidences were observed in the months March-May.

\section{Nosocomial vsRVd}

Seventeen of the 101 cases (17\%) were nosocomially acquired, of which 16 patients needed or were already in intensive care treatment during onset of RV symptoms. One child, a 4-monthold boy, was reported due to hypernatremia $>155 \mathrm{mmol} / \mathrm{L}$.

Fourteen patients were neonates in intensive care treatment. Three neonates were reported to have apneas as a sign of encephalopathy. A total of 8 neonates were diagnosed with necrotizing enterocolitis (NEC); in addition, NEC was suspected in 4 neonates who were treated with antibiotics and withdrawal of enteral nutrition. There was a significant male preponderance $(13 / 17 ; P=0.03)$.

Seven of the 16 neonates were reported by a single hospital between July and October 2009; 6 were preterm neonates (29-34 weeks of gestation) diagnosed with NEC and detection of both $\mathrm{RV}$ and adenovirus in feces. Additionally, this hospital reported a 2-month-old infant with tetralogy of Fallot and pyloric stenosis already in the ICU at the onset of RV disease.

Among the 17 reported nosocomial cases 1 death occurreda preterm neonate with 25 weeks of gestation. The child suffered

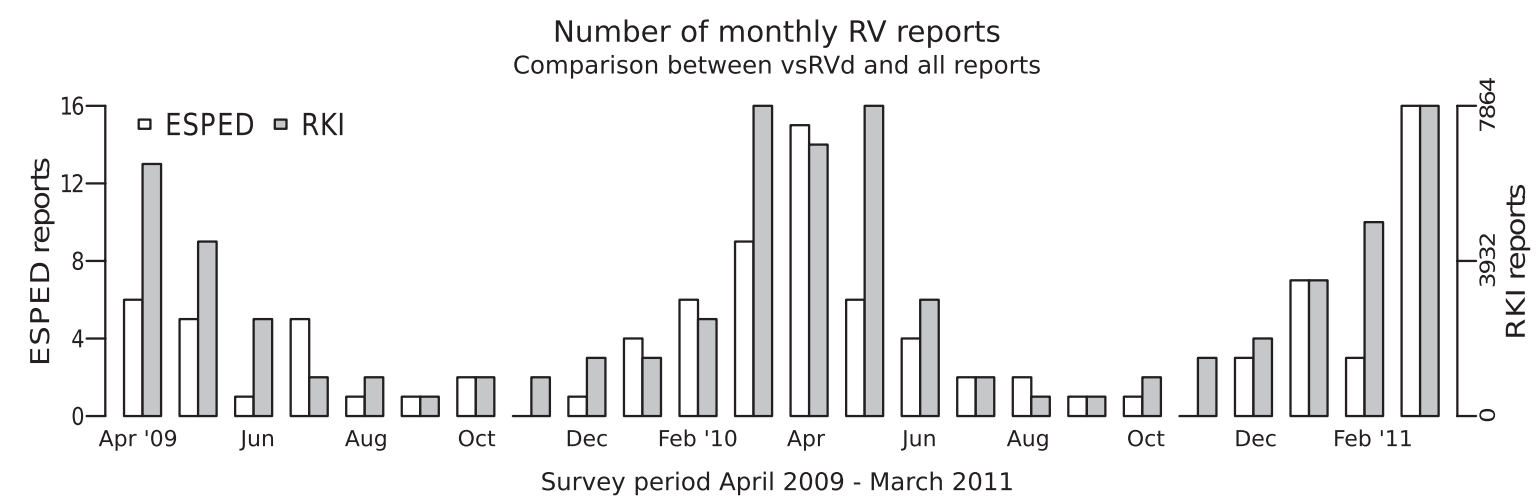

FIGURE 1. Monthly reports of RV disease. Left scale: ESPED reports (age group, 0-16 years). Right scale: All RV reports to the Robert Koch Institute (age group, 0-14 years). (Source of RKI data: with permission from Robert Koch-Institut: SurvStat, http:// www3.rki.de/SurvStat). 
TABLE 1. Descriptive Characteristics of the Patients With Community-acquired vsRVd Grouped by Inclusion Criteria (Multiple Entries per Patient Possible)

\begin{tabular}{|c|c|c|c|c|c|}
\hline & Total cohort & ICU & $\begin{array}{c}\text { Hypernatremia } \\
(>155 \mathrm{mmol} / \mathrm{L})\end{array}$ & $\begin{array}{l}\text { Hyponatremia } \\
(<125 \mathrm{mmol} / \mathrm{L})\end{array}$ & $\begin{array}{l}\text { Clinical signs of } \\
\text { encephalopathy }\end{array}$ \\
\hline $\mathrm{n}$ & 84 & 48 & 26 & 10 & 58 \\
\hline $\mathrm{n}$, male $(\%)$ & 45 & $29(60)$ & $13(50)$ & $5(50)$ & $31(53)$ \\
\hline Median age (mo) & 10.5 & 7 & 7 & 17.5 & 10.5 \\
\hline Median preadmission duration of symptoms (h) & 48 & 24 & 48 & 30 & 36 \\
\hline Median duration of hospitalization (days) & 6 & 8.5 & 6 & 6.5 & 6 \\
\hline
\end{tabular}

There was no significant statistical difference between median age and hypernatremia $(P=0.09)$ or hyponatremia $(P=0.12)$ compared with the median age of the patients without severe electrolyte disturbances.

a bilateral third-degree intraventricular hemorrhage with secondary hydrocephalus, which was treated with a Rickham reservoir. Additionally, the boy needed a surgical intervention due to a patent ductus arteriosus. After these procedures, he was in a stable condition. However, at the age of 2 months, he showed a rapid clinical deterioration with a suspected NEC and clinical and laboratory signs of septic shock (C-reactive protein, $107 \mathrm{mg} / \mathrm{L}$; interleukin-6 $>1000 \mathrm{pg} / \mathrm{mL}$ ), although no bacteria could be isolated in the cerebrospinal fluid (CSF) or blood cultures. The CSF contained 98 leukocytes $/ \mathrm{mL}$.

\section{Community-acquired vsRVd}

A total of 84 community-acquired cases were reported (45 male, 39 female). The median age was 10.5 months (IQR: 4.821.3 months; range: 0-109 months). The median preadmission duration of symptoms was 48 hours (IQR: 23.5-72 hours; range: 0 - 168 hours) and the median duration of hospital stay was 6 days (IQR: 5-11 days; range: 1-85 days). There were no relevant gender differences regarding patient age, preadmission duration of symptoms or duration of hospital stay. Seventy-nine of the 84 patients $(94 \%)$ were less than 5 years of age. The incidence in children less than 5 years of age in the various federal states ranged from 0 to $2.3 / 100,000 /$ year. The characteristics of the patients grouped by inclusion criteria are shown in Table 1 . The 2 oldest patients were a 8.6-year-old boy with a short bowel syndrome and need for total parenteral nutrition being admitted to the ICU due to gastrointestinal hemorrhage and a 9-year-old girl with an RV encephalitis without any known prior conditions.

\section{Intensive Care Treatment}

Forty-eight of the 84 patients (57\%) needed treatment in an ICU, and their median age ( 7 months) was significantly lower than those who were not admitted to the ICU (median age, 12.5 months; $P=0.01$ ). The hospital stay was significantly longer if the patient needed treatment in an ICU (median, 8.5 days versus 5 days in patients not treated in an ICU; $P<0.001)$.

\section{Electrolyte Disturbances}

In 26 of $84(31 \%)$ patients hypernatremia (>155 mmol/L) was observed, and $10(12 \%)$ patients had severe hyponatremia $(<125 \mathrm{mmol} / \mathrm{L})$. Patients with severe hypernatremia tended to be younger (median age, 8 months) compared with all other patients (median age, 12 months; $P=0.08$ ). Further details are listed in Table 1.

\section{Encephalopathies}

A total of 58 of $84(69 \%)$ patients had clinical signs of encephalopathy as defined by the inclusion criteria. Somnolence was reported in 44 patients. Five patients had apneas. Twenty-three patients suffered convulsions (multiple criteria for encephalopathy per patient possible). Most convulsions were associated with fever.
However, 5 of the seizures were afebrile and not associated with severe electrolyte disturbance or severe dehydration. Six of the 23 patients with convulsions also had very severe hypernatremia or hyponatremia ( $\mathrm{n}=2$ and $\mathrm{n}=4$, respectively).

Fourteen of the 58 patients with encephalopathy (24\%) had pathological electroencephalography. In 22 patients (38\% of the patients with encephalopathy, $61 \%$ of patients with convulsions), the CSF was examined revealing pathological findings in 6 patients: 5 patients had pleocytosis and were diagnosed with encephalitis. In a 9-year-old girl who had no convulsions but severe hyponatremia, somnolence and pathological electroencephalography (general deceleration), RV was identified in her CSF containing 2000 copies $/ \mathrm{mL}$ of RV. There was no pleocytosis or abnormal levels of glucose, protein or lactate. Magnetic resonance imaging on the day of admission first showed an increased signal intensity in the splenium of the corpus callosum whereas the control magnetic resonance imaging results showed signal changes in the cerebellum compatible with cerebellitis 5 days later. She was discharged with persisting neurologic symptoms. The CSF was tested for RV by polymerase chain reaction only in this one case. Of the remaining children with encephalopathy, 1 child was discharged with hemiparesis after an intraventricular hemorrhage, 1 child had residual convulsions and 1 child was discharged with developmental delay, although the delay was present before the RV disease. One patient was lost to follow-up after he was transferred to another hospital. The remaining patients had fully recovered at the time of hospital discharge.

\section{Clinical and Laboratory Findings}

Severe dehydration ( $>10 \%$ of the body weight) was observed in 39 patients; the median age of these patients ( 8 months) was significantly lower than those with a moderate or no dehydration (median age, 15.5 months; $P=0.04$ ). A synopsis of the laboratory findings is shown in Table 2. Fourteen of the 39 patients $(36 \%)$ with severe dehydration also presented with severe hypernatremia (>155 mmol/L); 6 of 39 patients (15\%) had severe hyponatremia $(<125 \mathrm{mmol} / \mathrm{L})$.

\section{Demographic Data}

Ethnicity-based data of the patients were obtained in 68 of the 84 reports of ambulatory-acquired RV disease. Fifty-four of the 68 patients (79\%) were Caucasians from Central Europe.

\section{Natural History and Deaths}

Seventy-one of the 84 patients (94\%) with communityacquired RV disease were classified as healthy at the time of discharge, 3 patients had residual gastrointestinal symptoms, 4 patients had persisting neurologic symptoms and 1 patient was discharged with a joint effusion, which was associated with a coinfection caused by Staphylococcus aureus. Discharge data were missing from 2 patients. 
TABLE 2. Descriptive Characteristics of the Laboratory Findings

\begin{tabular}{|c|c|c|c|c|c|c|c|}
\hline & WBC (/nL) & $\mathrm{CRP}(\mathrm{mg} / \mathrm{L})$ & $\mathrm{pH}$ & $\mathrm{BE}(\mathrm{mmol} / \mathrm{L})$ & $\mathrm{pCO}_{2}(\mathrm{~mm} \mathrm{Hg})$ & Bicarb.(mmol/L) & ALT (U/L) \\
\hline Range & 0.4 to 39.5 & 0 to 443 & 6.77 to 7.53 & -28.3 to 5.2 & 2.48 to 59.0 & 6.0 to 30.5 & 4 to 1364 \\
\hline IQR & 6.7 to 16.2 & 3 to 23.5 & 7.18 to 7.33 & -16.1 to -7.9 & 24.8 to 35.4 & 12.0 to 17.3 & 30 to 81 \\
\hline Median & 10.3 & 8.9 & 7.27 & -12.0 & 28.4 & 14.9 & 43 \\
\hline Mean & 12.3 & 27.9 & 7.24 & -12.0 & 28.8 & 14.8 & 123 \\
\hline
\end{tabular}

WBC indicates white blood cell count (normal values ( $\times 1000$ ): less than 2 years of age: $6.5-15.0,2-6$ years 5.0-12.0, 6-16 years 4.5-10.5); CRP, C-reactive protein (normal values $<5 \mathrm{mg} / \mathrm{L}$ ); $\mathrm{BE}$, base excess (normal values -2 to $+3 \mathrm{mmol} / \mathrm{L}$ ), $\mathrm{pCO}_{2}$, partial pressure of carbon dioxide (normal values $35-45 \mathrm{~mm} \mathrm{Hg}$ ), Bicarb., bicarbonate (normal values 21-26 mmol/L); ALT, alanine transaminase (normal values age $0-30$ days $<90 \mathrm{U} / \mathrm{L}, 1-12$ months $<80 \mathrm{U} / \mathrm{L}, 1-12$ years $<50 \mathrm{U} / \mathrm{L}$, $12-16$ years $<30 \mathrm{U} / \mathrm{L}$ ). (Normal values from Thomas, L.; Labor und Diagnose: Indikation und Bewertung von Laborbefunden für die medizinische Diagnostik, 7th ed., Frankfurt: TH Books-Verlags-Geschellschaft, 2008.)

Two deaths among the community-acquired vsRVd were reported. A 6-month-old infant with trisomy 21 with diarrhea beginning on the day of admission with fever and hyponatremia $(124 \mathrm{mmol} / \mathrm{L})$. RV and clostridium difficile toxin were identified in the stools. Hirschsprung disease was diagnosed during the acute exacerbation. The child died due to cardiac and respiratory failure and presumed septic shock, although the blood and CSF cultures were sterile.

A 12-month-old boy with symptoms of GE beginning 48 hours before hospital admission with severe dehydration $(>10 \%$ of the body weight) was admitted to the ICU during cardiopulmonary resuscitation. Maximum therapy with parenteral rehydration, mechanical ventilation and catecholamines was initiated but the boy died on the day of admission due to hypovolemic shock. The autopsy revealed no further or concomitant causes.

\section{DISCUSSION}

We present a 2-year prospective survey of vsRVd in children in Germany. Our estimation of the incidence of vsRVd is $1.2 / 100,000$ in children less than 5 years of age. This estimate is a minimum. The true incidence might be twice as high because ESPED does not capture all cases as shown by capture-recapture analyses. ${ }^{17,18}$ Comparing this incidence with the overall reported incidence of RV disease in the same age group in Germany $(115 / 10,000),{ }^{1}$ a vsRVd occurs in about $1 \%$ of all reported RV cases in children under 5 years of age. ${ }^{19}$ The median duration of hospitalization (6 days) is considerably longer than the median duration of all reported hospitalized RV cases (2-3 days). ${ }^{1}$

A recent retrospective analysis of the epidemiology of RV infections in children less than 5 years of age in Germany describing the burden of disease was recently published by Koch and Wiese-Posselt. ${ }^{1}$ This publication shows a detailed, descriptive analysis of national RV surveillance data collected in Germany between 2001 and 2008. Although epidemiologic data of hospitalizations and numbers of deaths are described in detail, the cases of vsRVd are not described or listed. The RV mortality rate we observed fits the data of this study that reported 8 deaths in children less than 5 years of age between 2004 and 2008. Another study estimated a death rate due to RV infections of 13 per year in children under the age of 5 years in Germany. ${ }^{4}$

Nosocomial RV infections cause a high disease burden. ${ }^{20-23}$ In particular, neonates are prone to severe illness. We observed 14 neonates treated in an ICU. There were significantly more male neonates with nosocomial vsRVd. Male preterm neonates had more complications and a poorer outcome than their female counterparts. ${ }^{24}$ This association may explain why male patients dominated this group.

$\mathrm{RV}$ in neonatal ICUs is a well-known problem, especially so, as the clinical signs in preterm neonates differ greatly from those in older children. ${ }^{25}$ In particular, diarrhea may not be the leading symptom. ${ }^{26,27}$ Most of the 14 neonates with nosocomially acquired
RV disease $(86 \%)$ were diagnosed with or had a suspected NEC. It has been reported that about $30 \%$ of NEC cases in preterm neonates are associated with $\mathrm{RV}^{28}$ The pathogenesis of this association remains unclear. It has been hypothesized that a viral nonstructural protein evokes abnormal intracellular calcium mobilization in enterocytes. ${ }^{29}$ There was a cluster of 6 neonates with NEC excreting $\mathrm{RV}$ and adenovirus reported by a single hospital over a period of 4 months. In that hospital, of course, nosocomial RV infection added seriously to the common neonatal pathology. ${ }^{25}$ Three neonates were reported to have apneas. Although this might be a clinical sign of encephalopathy, there were no data to distinguish the RV-associated apneas from apneas due to prematurity.

Ninety-four percent of patients with community-acquired RV disease were less than 5 years old. This is in accordance to epidemiologic findings in Germany and Europe. ${ }^{1,30}$

The overall median duration of admission was 6 days, which is considerably longer than the overall hospitalization duration of all acquired RV infections in children less than 5 years of age in Germany (2-3 days). ${ }^{1}$

The majority of patients with community-acquired vsRVd (69\%) had clinical signs of encephalopathy. The direct role of RV in these cases is not clear because neurologic symptoms may be unspecific and related to fever, dehydration or electrolyte disturbances. However, in 6 of these patients there was no severe dehydration, electrolyte disturbance or fever reported. Five children had afebrile seizures. One of them was coexcreting adenovirus, having severe hyponatremia at the time of admission as well. Afebrile convulsion associated with RV GE has been described numerous times. ${ }^{31-34}$

Although patients with RV GE and convulsions were included in this study, convulsions in patients with GE generally have a good prognosis. ${ }^{35}$ Thus, in most cases excessive diagnostic measures and therapeutic attempts can be avoided. ${ }^{36,37}$ Moreover, convulsions in case of GE are not a feature unique to RV. There are reports of other pathogens such as norovirus that are the primary cause of this clinical feature. ${ }^{38}$

The CSF was tested for RV only in 1 patient, containing 2000 copies $/ \mathrm{mL}$. The cranial magnetic resonance imaging of this patient showed signal intensity alterations in the cerebellum which appears to be a recurrent finding in patients with RV-associated cerebellitis. $^{39,40}$

Although the detection of RV in the CSF of patients with RV-associated encephalopathy may have no direct therapeutic or prognostic value, it should be considered if a lumbar puncture is performed due to other reasons because this might improve our understanding of the RV.

Severe hypernatremia $>155 \mathrm{mmol} / \mathrm{L}$ was observed in 26 patients. A recent retrospective study from Germany showed a significantly higher proportion of children with RV GE with hypernatremia $>150 \mathrm{mmol} / \mathrm{L}$ compared with RV negative GE cases, proposing hypernatremia to be a particular feature of RV. ${ }^{11}$ Another analysis from Sweden showed a hypernatremia in more than $9 \%$ of RV GE 
cases over a period of 11 years. ${ }^{41}$ The alanine transaminase was normal or slightly elevated in most cases. There have been observations of RV causing a moderate elevation of liver transaminase. ${ }^{42,43}$

RV vaccination is currently not generally recommended in Germany. However, health authorities of each federal state can establish local vaccination recommendations. In 5 federal states in Germany, the RV vaccine has been implemented in their local vaccination program (Saxony, Thuringia, Mecklenburg-Western Pomerania, Brandenburg and Schleswig-Holstein). In these states, the coverage is almost $60 \%$ as opposed to $20 \%$ in the 11 remaining federal states. ${ }^{44}$ There has been a significant drop in RV notifications in 2010 compared with 2006 in states with a high RV vaccination coverage ${ }^{44}$ Estimates show that a $90 \%$ RV vaccination coverage would significantly decrease disease burden in European regions. ${ }^{45,46}$ It has also been suggested that an RV vaccination coverage rate of about $90 \%$ might induce herd immunity, which may play a vital role in reducing the number of nosocomially acquired RV diseases.. ${ }^{47,48}$

A limitation of this study is that the survey began after the new RV vaccine was approved. As some federal states have already implemented the RV vaccine in their local vaccine recommendations, this may have caused a reduction in the number of vsRVd as the RV vaccine reduces the rate of RV hospitalizations. ${ }^{49}$

As detailed population data for 2011 were not available at the time of data retrieval from the German Federal Statistical Office, the estimation of incidence may be slightly inaccurate. No stool samples were collected to determine the RV serotype, as this might have increased the understanding of the RV virulence. It has been suggested that disease severity might vary with RV serotype.$^{50} \mathrm{RV}$ antigenemia might be observed during an acute RV GE as well as in asymptomatic children. ${ }^{51-53}$ Analysis of the patient serum might have contributed to determine the role of RV antigenemia in courses of vsRVd. Furthermore, a long-term follow-up of the patients might have given insight to the long-term effects of vsRVd.

Even in a country with guaranteed medical care for all and a high standard of pediatric care, vsRVd does occur. Although rare, vsRVd constitutes a considerable burden to the healthcare system and the families of the affected children.

\section{Conclusion}

Over a period of 2 years, 101 cases of vsRVd in Germany were reported. Although the incidence is relatively low compared with all RV cases, significant RV morbidity could be identified in a country with free access to health care for all and a highly developed healthcare system.

\section{ACKNOWLEDGMENTS}

The authors thank all participating clinics and Mrs. Heinrich (ESPED coordinator) for a successful collaboration.

\section{REFERENCES}

1. Koch J, Wiese-Posselt M. Epidemiology of rotavirus infections in children less than 5 years of age: Germany, 2001-2008. Pediatr Infect Dis J. 2011;30:112-117.

2. Parashar UD, Bresee JS, Gentsch JR, et al. Rotavirus. Emerg Infect Dis. 1998;4:561-570.

3. Tate JE, Burton AH, Boschi-Pinto C, et al. 2008 estimate of worldwide rotavirus-associated mortality in children younger than 5 years before the introduction of universal rotavirus vaccination programmes: a systematic review and meta-analysis. Lancet Infect Dis. 2012;12:136-141.

4. Soriano-Gabarró M, Mrukowicz J, Vesikari T, et al. Burden of rotavirus disease in European Union countries. Pediatr Infect Dis J. 2006;25:S7-S11.

5. Karsten C, Baumgarte S, Friedrich AW, et al. Incidence and risk factors for community-acquired acute gastroenteritis in north-west Germany in 2004. Eur J Clin Microbiol Infect Dis. 2009;28:935-943.
6. Soares-Weiser K, Maclehose H, Bergman H, et al. Vaccines for preventing rotavirus diarrhea: vaccines in use. Cochrane Database Syst Rev. 2012;2:CD008521.

7. Ruiz-Palacios GM, Pérez-Schael I, Velázquez FR, et al. Safety and efficacy of an attenuated vaccine against severe rotavirus gastroenteritis. $N$ Engl J Med. 2006;354:11-22.

8. Vesikari T, Matson DO, Dennehy P, et al. Safety and efficacy of a pentavalent human-bovine (WC3) reassortant rotavirus vaccine. $N$ Engl J Med. 2006;354:23-33

9. World Health Organization. WHO recommendation on global use of rotavirus vaccines. Wkly Epidemiol Rec. 2009;84:232-236.

10. Kobayashi S, Negishi Y, Ando N, et al. Two patients with acute rotavirus encephalitis associated with cerebellar signs and symptoms. Eur J Pediatr. 2010;169:1287-1291.

11. Kaiser P, Borte M, Zimmer KP, et al. Complications in hospitalized children with acute gastroenteritis caused by rotavirus: a retrospective analysis. Eur J Pediatr. 2012;171:337-345.

12. WeiB S, Streng A, Kries Rv, et al. Incidence of intussusception in early infancy: a capture-recapture estimate for Germany. Klin Padiatr. 2011;223:419-423.

13. Dressler F, Frosch M, Mönkemöller K, et al. Results of the German ESPEDrecording of new patients with juvenile dermatomyositis (JDM). Klin Padiatr. 2011;223:280-282.

14. Lainka E, Neudorf U, Lohse P, et al. Analysis of cryopyrin-associated periodic syndromes (CAPS) in German children: epidemiological, clinical and genetic characteristics. Klin Padiatr. 2010;222:356-361.

15. Göbel U, Heinrich B, Krauth KA, et al. [Process and outcome quality of the German Paediatric Surveillance Unit (ESPED)]. Klin Padiatr. 2010;222:92-97.

16. R Development Core Team. R: A Language and Environment for Statistical Computing; 2011.

17. von Kries R, Siedler A, Schmitt HJ, et al. Proportion of invasive pneumococcal infections in German children preventable by pneumococcal conjugate vaccines. Clin Infect Dis. 2000;31:482-487.

18. von Kries R, Heinrich B, Hermann, M. Pädiatrische Epidemiologie in Deutschland: Forschungsinstrument ESPED (Erhebungseinheit für seltene pädiatrische Erkrankungen in Deutschland). Monatsschr Kinderheilkd. 2001;149:1191-1197.

19. Van Damme P, Giaquinto C, Huet F, et al. Multicenter prospective study of the burden of rotavirus acute gastroenteritis in Europe, 2004-2005: the REVEAL study. J Infect Dis. 2007;195(suppl 1):S4-S16.

20. Gutiérrez-Gimeno MV, Martin-Moreno JM, Díez-Domingo J, et al. Nosocomial rotavirus gastroenteritis in Spain: a multicenter prospective study. Pediatr Infect Dis J. 2010;29:23-27.

21. Festini F, Cocchi P, Mambretti D, et al. Nosocomial Rotavirus Gastroenteritis in pediatric patients: a multi-center prospective cohort study. BMC Infect Dis. 2010;10:235.

22. Verhagen P, Moore D, Manges A, et al. Nosocomial rotavirus gastroenteritis in a Canadian paediatric hospital: incidence, disease burden and patients affected. J Hosp Infect. 2011;79:59-63.

23. Gleizes O, Desselberger U, Tatochenko V, et al. Nosocomial rotavirus infection in European countries: a review of the epidemiology, severity and economic burden of hospital-acquired rotavirus disease. Pediatr Infect Dis J. 2006;25(1 suppl):S12-S21.

24. Peacock JL, Marston L, Marlow N, et al. Neonatal and infant outcome in boys and girls born very prematurely. Pediatr Res. 2012;71:305-310.

25. Tai IC, Huang YC, Lien RI, et al. Clinical manifestations of a cluster of rotavirus infection in young infants hospitalized in neonatal care units. $J$ Microbiol Immunol Infect. 2012;45:15-21.

26. Sharma R, Hudak ML, Premachandra BR, et al. Clinical manifestations of rotavirus infection in the neonatal intensive care unit. Pediatr Infect Dis J. 2002;21:1099-1105

27. Verboon-Maciolek MA, Krediet TG, Gerards LJ, et al. Clinical and epidemiologic characteristics of viral infections in a neonatal intensive care unit during a 12-year period. Pediatr Infect Dis J. 2005;24:901-904.

28. Sharma R, Garrison RD, Tepas JJ 3rd, et al. Rotavirus-associated necrotizing enterocolitis: an insight into a potentially preventable disease? J Pediatr Surg. 2004;39:453-457.

29. Morris AP, Estes MK. Microbes and microbial toxins: paradigms for microbial-mucosal interactions. VIII. Pathological consequences of rotavirus infection and its enterotoxin. Am J Physiol Gastrointest Liver Physiol. 2001;281:G303-G310.

30. Forster J, Guarino A, Parez N, et al.; and the Rotavirus Study Group. Hospital-based surveillance to estimate the burden of rotavirus gastroenteritis 
among European children younger than 5 years of age. Pediatrics. 2009;123: e393-e400.

31. Komori H, Wada M, Eto M, et al. Benign convulsions with mild gastroenteritis: a report of 10 recent cases detailing clinical varieties. Brain Dev. 1995; 17:334-337.

32. Hung JJ, Wen HY, Yen MH, et al. Rotavirus gastroenteritis associated with afebrile convulsion in children: clinical analysis of 40 cases. Chang Gung Med J. 2003;26:654-659.

33. Narchi H. Benign afebrile cluster convulsions with gastroenteritis: an observational study. BMC Pediatr. 2004;4:2.

34. Lloyd MB, Lloyd JC, Gesteland PH, et al. Rotavirus gastroenteritis and seizures in young children. Pediatr Neurol. 2010;42:404-408.

35. Verrotti A, Nanni G, Agostinelli S, et al. Benign convulsions associated with mild gastroenteritis: a multicenter clinical study. Epilepsy Res. 2011;93:107-114.

36. Fasheh Youssef W, Pino Ramírez R, Campistol Plana J, et al. Benign afebrile convulsions in the course of mild acute gastroenteritis: a study of 28 patients and a literature review. Pediatr Emerg Care. 2011;27:1062-1064.

37. Durá-Travé T, Yoldi-Petri ME, Gallinas-Victoriano F, et al. Infantile convulsions with mild gastroenteritis: a retrospective study of 25 patients. Eur $J$ Neurol. 2011;18:273-278.

38. Chen SY, Tsai CN, Lai MW, et al. Norovirus infection as a cause of diarrheaassociated benign infantile seizures. Clin Infect Dis. 2009;48:849-855.

39. Takanashi J, Miyamoto T, Ando N, et al. Clinical and radiological features of rotavirus cerebellitis. AJNR Am J Neuroradiol. 2010;31:1591-1595.

40. Kubota T, Suzuki T, Kitase Y, et al. Chronological diffusion-weighted imaging changes and mutism in the course of rotavirus-associated acute cerebellitis/cerebellopathy concurrent with encephalitis/encephalopathy. Brain Dev. 2011;33:21-27.

41. Johansen K, Hedlund KO, Zweygberg-Wirgart B, et al. Complications attributable to rotavirus-induced diarrhoea in a Swedish paediatric population: report from an 11-year surveillance. Scand J Infect Dis. 2008;40:958-964.

42. Kovacs A, Chan L, Hotrakitya C, et al. Serum transaminase elevations in infants with rotavirus gastroenteritis. J Pediatr Gastroenterol Nutr. 1986;5:873-877.
43. Kawashima $\mathrm{H}$, Ishii $\mathrm{C}$, Ioi $\mathrm{H}$, et al. Transaminase in rotavirus gastroenteritis. Pediatr Int. 2012;54:86-88.

44. Uhlig U, Kostev K, Schuster V, et al. Rotavirus vaccination in Germany: analysis of nationwide surveillance data 2006 to 2010. Pediatr Infect Dis J. 2011;30:e244-e247.

45. Diez-Domingo J, Suriñach NL, Alcalde NM, et al. Burden of paediatric Rotavirus Gastroenteritis (RVGE) and potential benefits of a universal Rotavirus vaccination programme with a pentavalent vaccine in Spain. $B M C$ Public Health. 2010;10:469.

46. Panatto D, Amicizia D, Giacchino R, et al. Burden of rotavirus infections in Liguria, Northern Italy: hospitalisations and potential savings by vaccination. Eur J Clin Microbiol Infect Dis. 2011;30:957-964.

47. Paulke-Korinek M, Kundi M, Rendi-Wagner P, et al. Herd immunity after two years of the universal mass vaccination program against rotavirus gastroenteritis in Austria. Vaccine. 2011;29:2791-2796.

48. Buttery JP, Lambert SB, Grimwood K, et al. Reduction in rotavirus-associated acute gastroenteritis following introduction of rotavirus vaccine into Australia's National Childhood vaccine schedule. Pediatr Infect Dis J. 2011;30:S25-S29.

49. Castilla J, Beristain X, Martínez-Artola V, et al. Effectiveness of rotavirus vaccines in preventing cases and hospitalizations due to rotavirus gastroenteritis in Navarre, Spain. Vaccine. 2012;30:539-543.

50. Mota-Hernández F, Calva JJ, Gutiérrez-Camacho C, et al. Rotavirus diarrhea severity is related to the VP4 type in Mexican children. J Clin Microbiol. 2003;41:3158-3162.

51. Ramani S, Paul A, Saravanabavan A, et al. Rotavirus antigenemia in Indian children with rotavirus gastroenteritis and asymptomatic infections. Clin Infect Dis. 2010;51:1284-1289.

52. Moon S, Wang Y, Dennehy P, et al. Antigenemia, RNAemia, and innate immunity in children with acute rotavirus diarrhea. FEMS Immunol Med Microbiol. 2012;64:382-391.

53. Patel M, Rench MA, Boom JA, et al. Detection of rotavirus antigenemia in routinely obtained serum specimens to augment surveillance and vaccine effectiveness evaluations. Pediatr Infect Dis J. 2010;29:836-839. 